\title{
ACTIVIDAD ANTIINFLAMATORIA DEL EXTRACTO ETANÓLICO DE LAS HOJAS DE Manihot esculenta Crantz (YUCA) EN UN MODELO EXPERIMENTAL DE INFLAMACIÓN AGUDA
}

\author{
ANTI-INFLAMMATORY ACTIVITY OF THE ETHNOLIC EXTRACT OF THE LEAVES OF Manihot esculenta \\ Crantz (YUCA) IN AN EXPERIMENTAL MODEL OF ACUTE INFLAMMATION \\ Nathalie D. Amado-Cornejo ${ }^{1, a}$; Geraldine Atusparia-Flores ${ }^{1, a}$; Maricielo V. Huamán-Cabrera,a;



\begin{abstract}
RESUMEN
Introducción: Las hojas de Manihot esculenta Crantz (yuca) han sido usadas alrededor del mundo y a lo largo del tiempo con el fin de disminuir la respuesta antiinflamatoria. Objetivo: Evaluar la actividad antiinflamatoria del extracto etanólico de la hoja de Manihot esculenta crantz en ratas. Métodos: Se realizó un estudio tipo experimental. La población de estudio fueron 60 ratas albinas sp. Rattus novergicus, distribuidas en 4 grupos de 15 ratas cada uno. Se usó una solución homogénea de extracto etanólico de hojas de Manihot esculenta crantz para su administración vía intraperitoneal. Se utilizó carragenina como inductor inflamatorio, que fue administrado por vía intradérmica; para la medición del edema plantar, se hizo uso del Digital Water Plethysmometer (LE7500). Se administró al grupo control negativo solución de tween $80 /$ agua $(1: 10)$ a dosis de $1 \mathrm{ml} / 10 \mathrm{~g}$., al grupo control positivo betametasona $4 \mathrm{mg} / \mathrm{Kg}$ y a los grupos de tratamiento 1 y 2 Manihot esculenta crantz, $2 \mathrm{mg} / \mathrm{kg}$ y $4 \mathrm{mg} / \mathrm{kg}$, respectivamente. Se utilizó la prueba de ANOVA de 1 cola y la prueba post-hoc de Tukey, para las comparaciones entre los grupos. Resultados: En el 37,67\% del grupo de tratamiento 2 se observó una reducción del edema a las 3 horas de administrar Manihot esculenta crantz $(p<0,05)$. En otras mediciones se encontró una tendencia no significativa en ambos grupos de administración de Manihot esculenta crantz a la reducción del edema plantar. Conclusión: El extracto etanólico de Manihot sculenta a partir de la dosis de $4 \mathrm{mg} / \mathrm{kg}$ parece tener actividad antiinflamatoria en la reducción del edema plantar en el modelo animal utilizado.
\end{abstract}

Palabras clave: Manihot esculenta Crantz; Inflamación; Edema (fuente: DeCS BIREME).

\begin{abstract}
Introduction: Manihot esculenta Crantz (yucca) leaves have been used around the world and over time in order to decrease the anti-inflammatory response. Objective: To evaluate the anti-inflammatory activity of the ethanolic extract of the Manihot esculenta Crantz leaf in rats. Methods: An experimental study was conducted. The study population included 60 albino rats sp. Rattus novergicus, distributed in 4 groups of 15 rats each. A homogeneous solution of ethanolic extract of Manihot esculenta Crantz leaves was used for intraperitoneal administration. Carrageenan was used as an inflammatory inducer that was administered intradermally; for the measurement of plantar edema, the Digital Water Plethysmometer (LE7500) was used. Tween 80 / water solution (1:10) was administered to the negative control group at a dose of $1 \mathrm{~mL} . / 100 \mathrm{~g}$., To the betamethasone positive control group $4 \mathrm{mg} / \mathrm{Kg}$ and to treatment groups 1 and 2 Manihot esculenta Crantz, $2 \mathrm{mg} / \mathrm{kg}$ and $4 \mathrm{mg} / \mathrm{kg}$, respectively. The 1-tail ANOVA test and the Tukey post hoc test were used for comparisons between the groups. Results: In $37.67 \%$ of treatment group 2 a reduction in edema was observed 3 hours after administering Manihot esculenta Crantz $(p<0.05)$. In both administration groups of Manihot esculenta Crantz there was a nonsignificant trend to reduce plantar edema with values close to significance. Conclusion: The Manihot esculenta Crantz ethanolic extract at a $4 \mathrm{mg} / \mathrm{kg}$ dose probably have anti-inflammatory activity in this animal model of acute inflamation.
\end{abstract}

Key words: Manihot esculenta Crantz; Inflammation; Edema (source: MeSH NLM).

\footnotetext{
${ }^{1}$ Facultad de Medicina Humana, Universidad Ricardo Palma, Lima-Perú.

2 Instituto de Investigación en Ciencias Biomédicas, Universidad Ricardo Palma, Lima-Perú.

a Estudiante de medicina.

${ }^{b}$ Master en bioquímica.

Química farmacéutica.

Citar como: Nathalie D. Amado-Cornejo, Geraldine Atusparia-Flores, Maricielo V Huamán-Cabrera, Ángela Méndez-Pajares, Etty Prado-Asencios, Hilda Jurupe-Chico, Vilma Herencia-Reyes. Actividad antiinflamatoria del extracto etanólico de las hojas de Manihot esculenta Crantz (yuca) en un modelo experimental de inflamación aguda Rev. Fac. Med. Hum. Enero 2020; 20(1):94-98. DOI 10.25176/RFMH.v20i1.2552 


\section{INTRODUCCIÓN}

La inflamación es una respuesta protectora del organismo para librarlo del agente causante de una lesión, las cuales pueden ser microrganismos patógenos, células necróticas e incluso la hipoxia. La inflamación puede ser aguda o crónica en función de la naturaleza del estímulo y la eficacia de la reacción inicial para eliminarlo. La fase aguda se inicia de forma rápida (en minutos) y dura poco, se caracteriza por la exudación de líquido y proteínas plasmáticas (edema) y la migración de neutrófilos. La fase crónica se caracteriza por la presencia de linfocitos, macrófagos, proliferación vascular, fibrosis y destrucción tisular. La inflamación termina cuando se elimina el agente responsable del daño(1).

Para combatir la inflamación, principalmente por sus efectos no deseados como el dolor, el tumor u otros, es común el uso de fármacos antiinflamatorios no esteroideos (AINES) y glucocorticoides, que son fármacos que pueden ocasionar reacciones adversas, en el caso de los AINES producen efectos sobre la función gastrointestinal, cardiovascular y renal; y con respecto a los glucocorticoides sobre la función endocrina y metabólica, musculoesquelética, inmunitaria, gastrointestinal, oftalmológica y del sistema nervioso central(2).

Debido a esto, en la actualidad los tratamientos buscan disminuir los riesgos potenciando los beneficios y dentro de ellos encontramos alternativas terapeúticas como los productos naturales; dentro de estos destaca el uso de plantas como alternativa o coadyudante en los tratamientos. Tal es el caso de Manihot esculenta Crantz (MEC) "YUCA" también llamada: mandioca, tapioca, shushu, muk shue, cassave, imanoka, maniba, kasaba, katela boodin, árbol de batata y arrurruz brasileño(3).

La MEC, es un arbusto leñoso de la familia Euphorbiaceae, de la subfamilia Crotonoideae se cultiva ampliamente en los países tropicales y subtropicales de África, Asia y América Latina, con una producción estimada de 276,7 millones de toneladas. Se clasifica como el cuarto cultivo más importante del mundo y un alimento básico para casi mil millones de personas ${ }^{(3,4)}$. Al estudiarse, se contribuirá con la investigación de plantas medicinales, de las cuales nuestro país tiene una gran diversidad ${ }^{(5)}$.

A pesar de que contiene algunas sustancias tóxicas, asociadas con la alta concentración de glucósidos cianogénicos como la linamarina, lotaustralina y lataustralina ${ }^{(3,8)}$, que varían según la ubicación, los cultivares, las condiciones ambientales y el estado de nutrientes del suelo(4). La planta almacena una serie de compuestos beneficiosos como antioxidantes, vitamina A, B1, B2, C, hierro, zinc, manganeso, magnesio, calcio, fósforo, potasio, zinc, cobre, sodio, antocianinas (flavonoides), saponinas, esteroides y glucósidos. Estudios previos han informado que varias plantas que contienen saponina y flavonoides poseen efectos analgésicos, antiinflamatorios, antipiréticos, antimicrobianos y anticancerigenos ${ }^{(4,6-8)}$.

Debido a esto, es usado en Nigeria para el tratamiento de la tiña, el tumor, la conjuntivitis, las llagas, los abscesos, los forúnculos, la prostatitis, el espasmo, el reumatismo, la fiebre, el dolor de cabeza, la diarrea, el marasmo, la pérdida del apetito y la mordedura de serpiente ${ }^{(3)}$.

Por lo tanto, en el presente estudio de tipo experimental, buscamos evaluar la actividad antiinflamatoria del extracto etanólico de la hoja de Manihot esculenta Crantz (EEHMEC) en ratas, utilizando modelos experimentales validados para la inducción de inflamación aguda.

\section{MÉTODOS}

Se realizó un estudio experimental en un modelo animal de inflamación aguda.

Preparación del material vegetal: Se recolectó el material vegetal elegido, MEC (YUCA), desde la selva central, enviando la muestra para su respectiva posición taxonómica, según el sistema de clasificación de Cronquist (1998) realizado en el Museo de Historia Natural - Universidad Mayor de San Marcos (UNMSM).

Se obtuvo una muestra completa de hojas y tallos; se dejaron al secado a $40^{\circ} \mathrm{C}$ en horno por 3 días y luego a temperatura ambiente bajo sombra por 3 días. Se trituraron manualmente, la molienda se realizó con la ayuda de un mortero y un rodillo.

Luego, el material vegetal ya en polvo y bajo condiciones de calidad de la muestra según las normas establecidas por farmacopeas, se colocó en una solución alcoholica de 96\% macerándose por 7 días, luego se filtró, llevado al secado por 10 días a temperatura y humedad ambiental obteniendo el extracto seco y se preparó una solución usando como diluyente tween 80/agua (1:10), logrando una solución homogénea para su administración vía intraperitoneal (IP).

Preparación del material animal: Se utilizó una muestra de 60 ratas albinas sp. Rattus novergicus, jóvenes de un mes y medio de edad, cuyos pesos 
aproximadamente eran de $150 \mathrm{~g}$. Adquiridas en el Centro Nacional de Productos Biológicos del Instituto Nacional de Salud - Ministerio de Salud (MINSA), distribuidas de manera aleatoria en cuatro grupos: Grupo control negativo, grupo control positivo y dos grupos tratamiento, de 15 ratas cada uno. Se mantuvieron a temperatura y humedad ambiental con acceso libre al aire, alimentación balanceada y agua ad libitum por 8 días para su aclimatación en el Laboratorio de Farmacología de la Facultad de Medicina de la Universidad Ricardo Palma.

\section{Preparación del modeloexperimentalfarmacológico:}

Se procedió a administrar vía intraperitoneal material biológico a cuatro grupos experimentales: 1. Grupo control negativo $(\mathrm{CN})$ : solución de tween 80/agua (1:10) a dosis de $1 \mathrm{ml} / 100 \mathrm{~g}$., 2 . Grupo control positivo $(\mathrm{CP})$ : betametasona $4 \mathrm{mg} / \mathrm{Kg}$. 3. Grupo tratamiento 1 (GT1): solución del EEHMEC a dosis de $2 \mathrm{mg} / \mathrm{kg}$ y Grupo tratamiento 2 (GT2) solución del EEHMEC a dosis de $4 \mathrm{mg} / \mathrm{kg}$.

El modelo experimental se inicia con la toma de datos basales como la medición del volumen de la pata trasera derecha en el Digital Water Plethysmometer (LE7500) continuando con la administración de las soluciones a los cuatro grupos experimentales: $\mathrm{CN}$, CP, GT1 Y GT2, luego de 30 minutos se les realizó la inducción de la inflamación. La inducción de la inflamación se realizó mediante el test de edema plantar inducido por carragenina, administrando en la aponeurosis plantar de la pata trasera derecha de la rata vía intradérmica $0,1 \mathrm{ml}$ de carragenina $1 \%$.

Luego de la inducción de la inflamación se procedió a medir el volumen de la pata trasera derecha, a los 30 minutos, 1, 2, 3, 4, 5 y 24 horas siempre utilizando el Digital Water Plethysmometer (LE7500) para obtener los resultados.

Los resultados obtenidos se colocaron en una base de datos realizada en Microsoft Excel 2010 y procesados en el programa estadístico SPSS 22. Se utilizó la prueba de ANOVA de 1 cola y la prueba post hoc de Tukey, para las comparaciones entre los grupos. Se trabajó a una significancia estadística de un valor de $p<0,05$ y un intervalo de confianza al $95 \%$.

\section{RESULTADOS}

El EEMEC a dosis de $4 \mathrm{mg} / \mathrm{kg}$ produjo una reducción significativa $(p<0,05)$ del edema a las 3 horas en un $37,67 \%$ en comparación al control negativo, además el control positivo también produjo una reducción significativa $(p<0,05)$ del edema a las 4 y 5 horas de administrado. No hubo diferencias estadísticamente significativas en el resto de horas con respecto al control negativo, como se observa en la tabla 1.

Tabla 1. Diferencias en el incremento del edema plantar inducido por carragenina según la administración de los distintos tratamientos.

\begin{tabular}{|c|c|c|c|c|c|c|c|c|}
\hline \multirow{2}{*}{ Tratamiento } & \multirow{2}{*}{$\begin{array}{c}\text { Dosis } \\
\text { (mg/ } \\
\text { kg) }\end{array}$} & \multicolumn{7}{|c|}{ Tiempo (h) } \\
\hline & & 0,5 & 1 & 2 & 3 & 4 & 5 & 24 \\
\hline $\begin{array}{l}\text { Control Negativo: } \\
\text { Suero fisiológico }\end{array}$ & $\begin{array}{c}10 \mathrm{ml} / \\
\mathrm{kg}\end{array}$ & $0.25 \pm 0.11$ & $0.34 \pm 0.25$ & $0.90 \pm 0.21$ & $1.46 \pm 0.26$ & $1.22 \pm 0.42$ & $1.16 \pm 0.37$ & $0.66 \pm 0.33$ \\
\hline $\begin{array}{l}\text { Control Positivo: } \\
\text { Betametasona }\end{array}$ & $4 \mathrm{mg} / \mathrm{kg}$ & $\begin{array}{c}0.38 \pm 0.23 \\
(-52 \%)^{* *}\end{array}$ & $\begin{array}{r}0.42 \pm 0.17 \\
(-23.52 \%)\end{array}$ & $\begin{array}{c}0.53 \pm 0.24 \\
(41.11 \%)\end{array}$ & $\begin{array}{c}0.96 \pm 0.25 \\
(34.24 \%)\end{array}$ & $\begin{array}{c}0.62 \pm 0.22^{*} \\
(49.18 \%)\end{array}$ & $\begin{array}{c}0.61 \pm 0.22^{*} \\
(47.41 \%)\end{array}$ & $\begin{array}{c}0.38 \pm 0.19 \\
(42.42 \%)\end{array}$ \\
\hline $\begin{array}{l}\text { Tratamiento: } \\
\text { Solución }\end{array}$ & $2 \mathrm{mg} / \mathrm{kg}$ & $\begin{array}{c}0.20 \pm 0.28 \\
(20 \%)\end{array}$ & $\begin{array}{c}0.49 \pm 0.32 \\
(-44.11 \%)\end{array}$ & $\begin{array}{c}1.00 \pm 0.58 \\
(-11.11 \%)\end{array}$ & $\begin{array}{c}0.97 \pm 0.42 \\
(33.56 \%)\end{array}$ & $\begin{array}{c}0.90 \pm 0.36 \\
(26.22 \%)\end{array}$ & $\begin{array}{c}1.02 \pm 0.34 \\
(12.06 \%)\end{array}$ & $\begin{array}{c}0.49 \pm 0.29 \\
(25.75 \%)\end{array}$ \\
\hline $\begin{array}{l}\text { de Manihot } \\
\text { esculenta Crantz }\end{array}$ & $4 \mathrm{mg} / \mathrm{kg}$ & $\begin{array}{c}0.13 \pm 0.19 \\
(48 \%)\end{array}$ & $\begin{array}{c}0.55 \pm 0.54 \\
(-61.76 \%)\end{array}$ & $\begin{array}{c}0.84 \pm 0.44 \\
(6.66 \%)\end{array}$ & $\begin{array}{c}0.91 \pm \\
0.51 * \\
(37.67 \%)\end{array}$ & $\begin{array}{c}0.81 \pm 0.48 \\
(33.60 \%)\end{array}$ & $\begin{array}{c}0.79 \pm 0.45 \\
(31.89 \%)\end{array}$ & $\begin{array}{c}0.42 \pm 0.34 \\
(36.36 \%)\end{array}$ \\
\hline
\end{tabular}

* La reducción del edema se diferencia de forma estadísticamente significativa $(p<0,05)$ comparado al control.

**Los valores entre paréntesis son el porcentaje de inhibición del edema calculado relativo al control. 
Así mismo, se observa una tendencia a la reducción del edema plantar con la dosis de $4 \mathrm{mg} / \mathrm{kg}$ con valores cercanos a la significancia no solo a las 3 horas de administración.

\section{DISCUSIÓN}

Los resultados del presente estudio indican que el EEHMEC, administrado vía intraperitoneal presenta efectos antiinflamatorios significativos a las 3 horas de administración siendo estos de corta duración. Estos resultados fueron similares a los hallados por Bokanisereme et al, quienes administraron tres diferentes dosis (100, 250 y $500 \mathrm{mg} / \mathrm{kg}$ ) EEHMEC por vía oral a ratas. Comparado con el control negativo (suero fisiológico) encontró una disminución significativa $(p<0,001)$ del edema plantar a las $5 \mathrm{hrs}$ posteriores a su administración. La dosis de $100 \mathrm{mg} / \mathrm{kg}$ generó una disminución del edema $(26,2 \%)$ superior a las otras dosis e incluso al control positivo (indometacina). Sin embargo, no hubo respuesta terapeútica significativa en las horas previas. Nuestros resultados evidencian diferencias con los resultados encontrados por Bokanisereme et al., ya que la disminución del edema plantar se observó a las 3 horas siendo de corta duración ${ }^{(9)}$.

Así también, estos resultados coinciden con los reportados por 0 . 0 . Adeyemi et al, quienes más bien utilizaron un extracto acuoso de hojas de Manihot sculenta que administraron a tres diferentes dosis (100, 200 y $400 \mathrm{mg} / \mathrm{kg}$ ) por vía oral a ratas y como instrumento de medición del diámetro del edema plantar en las ratas hicieron uso de un hilo blanco. Ellos evidenciaron una reducción significativa $(p<0,05)$ del edema comparado al control negativo (suero fisiológico) desde las 2 a 24 horas posteriores a la aplicación de carragenina. La máxima respuesta terapeútica fue obtenida con la dosis más alta $(400 \mathrm{mg} / \mathrm{kg}$ ) y fue superior a la del control positivo (indometacina). Comparado con nuestra investigación, los resultados antiinflamatorios difieren en cuanto a periodo de latencia y duración, esto es atribuible al tipo de extracto pero a la vez resulta contradictorio por ser un extracto acuoso frente al extracto etanolico ${ }^{(10)}$.

A diferencia de estas investigaciones previas en las que se utilizó un extracto acuoso, se preparó un extracto etanólico, porque el procesamiento inadecuado de las hojas de MEC genera que haya altas concentraciones de cianuro y gran pérdida del contenido de proteínas y vitaminas. En la preparación de un extracto acuoso se hierven las hojas de MEC en agua lo que permite eliminar todos los cianógenos, pero genera grandes pérdidas de proteínas, vitaminas C, tiamina, riboflavina y ácido nicotínico que son sustancias que probablemente tengan un papel fundamental en la actividad terapeútica del extracto. En este trabajo se trituraron las hojas para que las enzimas entren en contacto y catalicen la hidrólisis de los cianógenos, además de darles un periodo de reposo durante 72 horas a $40^{\circ} \mathrm{C}$ para permitir que la enzima linamirasa hidrolice la linamarina a cianhidrina de acetona y a la hidroxinitriloliasa para hidrolizar cianhidrina de acetona a cianuro de hidrógeno, que escapa como gas. En la preparación del extracto etanolico, las hojas de MEC se trataron con rigurosidad, sin embargo, los resultados obtenidos con dicho extracto no lograron el efecto antiinflamatorio que se evidenciaron en las investigaciones de Bokanisereme et al y O. O. Adeyemi et $\mathrm{al}^{(9-12)}$.

Se utilizó la vía de administración intraperitoneal porque permite una rápida y notable absorción de medicamentos debido a que la cavidad peritoneal posee una circulación linfática que drena directamente tanto a la circulación portal como la sistémica ${ }^{(13)}$. Así también, la carragenina es un inductor de inflamación aguda local por tanto el efecto antiinflamatorio máximo que se evidenció en el estudio fue sobre la fase tardía de dicha inflamación, esta fase se caracteriza por ser dependiente del calcio y de la óxido nítrico sintasa ${ }^{(14)}$. Es por esto que el probable mecanismo de acción por el que las hojas de extracto etanolico Manihot esculenta Crantz hayan producido su efecto antiinflamatorio fuera mediado por la inhibición en la formación de eicosanoides al actuar como un inhibidor de la enzima óxido nítrico sintetasa durante la fase tardía de la inflamación aguda por los resultados obtenidos en nuestro modelo experimental como en investigaciones previas.

Entre las limitaciones del estudio se encuentra la posibilidad de error aleatorio y el consecuente riesgo de asociaciones espúreas debido al efecto de realizar múltiples comparaciones, por lo que es necesario estudios en otros modelos animales a fin de corroborar nuestros hallazgos.

\section{CONCLUSIÓN}

El extracto etanólico de Manihot esculenta Crantz a partir de la dosis de $4 \mathrm{mg} / \mathrm{kg}$ muestra un efecto sobre la inflamación aguda al reducir el edema plantar significativamente. Se recomienda realizar estudios utilizando modelos de inflamación crónica y mayores dosis del extracto de esta planta a fin de definir su potencial terapéutico. 
Contribuciones de autoría: Los autores participaron en la génesis de la idea, diseño de proyecto, recolección e interpretación de datos, análisis de resultados, preparación del manuscrito.

Financiamiento: Financiado por el fondo de investigación de la URP (A.C.U. 0957-2017).
Conflicto de interés: Los autores declaran no tener ningún conflicto de interés.

Recibido: 10 de mayo 2019

Aprobado: 10 de junio 2019

Correspondencia: Vilma Herencia Reyes.

Dirección: Laboratorio de Farmacología FAMURP, I-304. Avenida Benavides 5440, Santiago de Surco, Lima-Perú. Teléfono: (01) 708-0000 Anexo: 6010

Correo: vilma.herencia@urp.edu.pe

\section{REFERENCIAS BIBLIOGRÁFICAS}

1. Robbins SL, Kumar V, Abbas AK, Aster JC, Cotran RS, Perkins JA et al. Robbins y Cotran: patología estructural y funcional. 2015 Disponible en: http://www.sidalc.net/cgi-bin/wxis.exe/?IsisScript=UCC xis\&method=post\&formato=2\&cantidad=1\&expresion $=\mathrm{mfn}=099790$

2. Lorenzo-Velázquez B, Lorenzo Fernández $\mathrm{P}$ de. Manual de farmacología básica y clínica. Madrid: Editorial Médica Panamericana; 2013. Disponble en: https://www.medicapanamericana.com/Libros/Libro/3987/Velazquez Farmacologia-Basica-y-Clinica.html

3. Bahekar S, Kale R. Phytopharmacological aspects of Manihot Sculenta Crantz (Cassava) - A review. Mintage J Pharm Med Sci. 2(1):4-5. Disponible en: https:// www.researchgate.net/publication/315766976 Phytopharmacological aspects of Manihot esculenta_CrantzCassava-A Review

4. Latif S, Müller J. Potential of cassava leaves in human nutrition: A review. Trends Food Sci Technol. agosto de 2015;44(2):147-58. Disponible en: https:// pubag.nal.usda.gov/catalog/5427736

5. Brack Egg A. Biodiversidad: firmeza necesaria [Internet]. Red Voltaire. 2005 [citado el 14 de junio de 2019]. Disponible en: https://www.voltairenet.org/ article128871.htm

6. Miladiyah I, Dayi F, Desrini S. Analgesic activity of ethanolic extract of Manihot esculenta Crantz leaves in mice. Universa Med. 2011;30(1):3-10. DOI: 10.18051/UnivMed.2011.v30.3-10

7. Quartey $\mathrm{E}$, Amoatey $\mathrm{H}$, Achoribo $\mathrm{E}$, Owusu-Ansah M, Nunekpeku W, Donkor $\mathrm{S}$, et al. Phytochemical Constituents and Antioxidant Activities in Leaves of 14 Breeding Lines of Cassava (Manihot esculenta Crantz). Am J Exp Agric. el 10 de enero de 2016;12(5):1-10. DOI: 10.9734/AJEA/2016/18087
8. Ajayi El, Agarwal A, Banerjee UC, Olorunsogo OO. Ethanol extract of Manihot esculenta leaf: A potential source of antioxidant, xanthine oxidase and lipase inhibitors. Analele Stiintifice Ale Univ Alexandru loan Cuza Din lasi Sec II Genet Si Biol Mol. 2017;18(1):17-23.

9. Bokanisereme UFY, Okechukwu PN. Anti-inflammatory, analgesic and antipyretic activity of cassava leaves extract. Asian J Pharm Clin Res. 2013;6:89-92. Disponible en: https://www.researchgate.net/publication/287283103

10. Adeyemi OO, Yemitan OK, Afolabi L. Inhibition of chemically induced inflammation and pain by orally and topically administered leaf extract of Manihot esculenta Crantz in rodents. J Ethnopharmacol. 2 de septiembre de 2008;119(1):6-11. Disponible en: https://doi.org/10.1016/j.jep.2008.05.019

11. Bradbury JH, Denton IC. Mild method for removal of cyanogens from cassava leaves with retention of vitamins and protein. Food Chem septiembre de 2014;158:417-20. Disponible en: https://doi.org/10.1016/j. foodchem.2014.02.132

12. Ferraro V, Piccirillo $C$, Tomlins $\mathrm{K}$, Pintado ME. Cassava (Manihot esculenta Crantz) and Yam (Dioscorea spp.) Crops and Their Derived Foodstuffs: Safety, Security and Nutritional Value. Crit Rev Food Sci Nutr. el 9 de diciembre de 2016;56(16):2714-27. Disponible en: https://doi.org/10.1080/10408398.2014 .922045

13. Montenegro J, Correa Rotter R, Riella MC. Tratado de diálisis peritoneal. Barcelona: Elsevier; 2016

14. Handy RLC, Moore PK. A comparison of the effects of L-NAME, 7-NI and L-NIL on carrageenan-induced hindpaw oedema and NOS activity. Br J Pharmacol. marzo de 1998;123(6):1119-26. DOI: 10.1038/sj.bjp.0701735

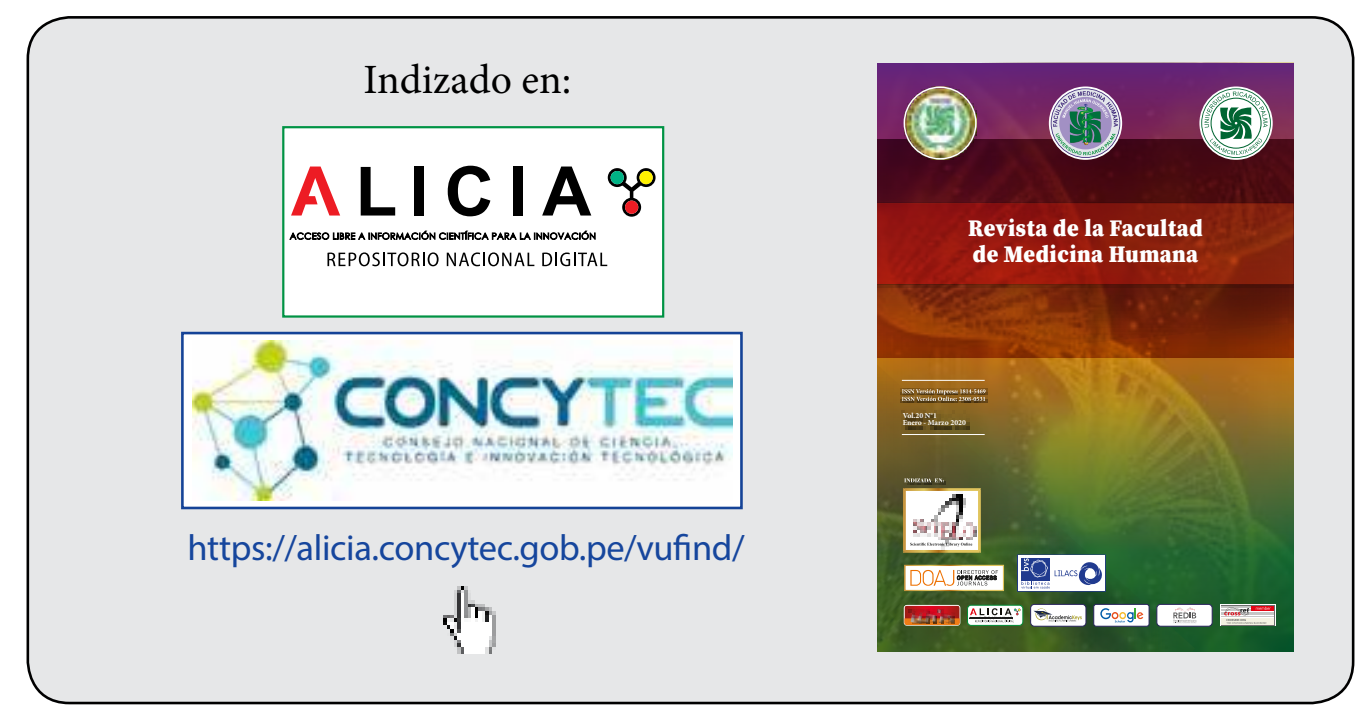

